\title{
Utilising proteomic approaches to understand oncogenic human herpesviruses (Review)
}

\author{
CHRISTOPHER B. OWEN, DAVID J. HUGHES, BELINDA BAQUERO-PEREZ, ANJA BERNDT, \\ SOPHIE SCHUMANN, BRIAN R. JACKSON and ADRIAN WHITEHOUSE
}

\begin{abstract}
School of Molecular and Cellular Biology and Astbury Centre for Structural Molecular Biology, Faculty of Biological Sciences, University of Leeds, Leeds, LS2 9JT, UK
\end{abstract}

Received May 23, 2014; Accepted June 10, 2014

DOI: $10.3892 / \mathrm{mco} .2014 .341$

\begin{abstract}
The $\gamma$-herpesviruses Epstein-Barr virus and Kaposi's sarcoma-associated herpesvirus are successful pathogens, each infecting a large proportion of the human population. These viruses persist for the life of the host and may each contribute to a number of malignancies, for which there are currently no cures. Large-scale proteomic-based approaches provide an excellent means of increasing the collective understanding of the proteomes of these complex viruses and elucidating their numerous interactions within the infected host cell. These large-scale studies are important for the identification of the intricacies of viral infection and the development of novel therapeutics against these two important pathogens.
\end{abstract}

\section{Contents}

1. Introduction

2. Approaches to proteomics

3. Understanding $\gamma$-herpesviruses through proteomics

4. Combining OMICS approaches to maximize function

5. Future perspectives

\section{Introduction}

The Epstein-Barr virus (EBV), also referred to as human herpesvirus 4 (HHV-4), was the first human oncogenic virus to be isolated 50 years ago by Epstein and co-workers from lymphoblasts cultured from a Burkitt's lymphoma (1). Thirty

Correspondence to: Professor Adrian Whitehouse, School of Molecular and Cellular Biology and Astbury Centre for Structural Molecular Biology, Faculty of Biological Sciences, University of Leeds, Leeds, LS2 9JT, UK

E-mail: a.whitehouse@leeds.ac.uk

Key words: proteomics, mass spectrometry, $\gamma$-herpesvirus years later, herpesviral DNA was identified in cells of Kaposi's sarcoma (KS), giving rise to a new virus, $\mathrm{KS}$-associated herpesvirus (KSHV), also referred to as HHV-8 (2). KSHV was shown to be highly related to EBV and, therefore, represented the second human $\gamma$-herpesvirus. To date, these are the only known oncogenic human herpesviruses and are each associated with numerous malignancies (Table I).

Over $90 \%$ of the global adult population is seropositive for EBV and infection usually occurs asymptomatically in early childhood $(3,4)$. However, primary infection during adolescence often results in infectious mononucleosis, which is characterised by $\mathrm{T}$-cell hyperproliferation with a strong $\mathrm{CD}^{+}$ response, with affected individuals suffering from flu-like symptoms and severe fatigue. Furthermore, only a minute proportion of those infected with EBV develop malignant diseases (5) and these diseases are invariably associated with immune suppression, either iatrogenic, or due to human immunodeficiency virus (HIV) or malaria co-infection (6).

The KSHV prevalence in northern Europe is significantly lower compared to that of EBV, with approximately $3 \%$ of the population testing seropositive $(7,8)$. However, several parts of Africa have an incidence rate of $>80 \%$, whereas intermediate rates are found in the Mediterranean $(9,10)$. Concurrent with these findings, $\mathrm{KS}$ is one of the most common cancers in sub-Saharan Africa, where individuals are often co-infected with KSHV and HIV (11).

The two $\gamma$-herpesviruses readily infect B lymphocytes, where they establish a lifelong reservoir $(12,13)$. However, initial infection and replication may also occur in oral epithelial cells, allowing transmission of the viruses via contaminated saliva (12-17). Similar to all members of the herpesvirus family, EBV and KSHV have a biphasic life cycle, consisting of a latent phase and a lytic replication cycle (18). Following primary infection, viral latency is established with the viral genome maintained as a circularised episome in the nucleus of the host cell. During this phase of infection, no infectious virions are produced. A limited number of genes are expressed during latency, encoding proteins that promote cell survival and proliferation, in order to avoid loss of the viral episome. While this proliferation is limited by the immune system in healthy individuals, individuals with a compromised immune system, under conditions such as iatrogenic immunosuppression, suffering from congenital immunodeficiency 
or co-infected with HIV, may develop lymphoproliferative disorders (19).

EBV latency is associated with at least four gene expression programmes, termed latency 0, I, II and III. Latency III, which is found in the B cells of post-transplant lymphoproliferative disease (PTLD) patients and in in vitro immortalised B cells, displays the broadest gene expression profile, with expression of all the EBV-encoded nuclear antigens (EBNA1, 2, 3A, $3 \mathrm{~B}, 3 \mathrm{C}$ and leader protein), three latent membrane proteins (LMP1, 2A and 2B) and several non-coding RNAs, such as the Epstein-Barr virus-encoded small RNAs (EBERs) and microRNAs (miRNAs) $(5,20,21)$. Latency II, which is observed in Hodgkin's lymphoma and EBV-associated carcinomas, is more restrictive, as only EBNA1 and the LMPs are expressed (along with EBERs). Latency I, the most restrictive latency programme associated with malignancy is found in Burkitt's lymphoma, where, along with the EBERs, EBNA1 is the only EBV protein expressed. However, in resting memory B cells, the latent reservoir in immunocompetent individuals, no viral proteins are expressed, which is known as latency $0(22,23)$.

It is considered that primary infection by EBV initially requires the latency III transcriptional programme in order to drive the proliferation and differentiation of $\mathrm{B}$ cells to promote host colonisation. However, as several of the EBNAs are immunodominant, a healthy immune system prevents this from evolving into a lymphoproliferative disorder. Therefore, EBV possesses various sophisticated mechanisms that promote the downregulation of gene expression as latency is established. These mechanisms are poorly understood, but it is hypothesized that EBV transcription is sequentially restricted as it transits the germinal centre reaction on its way to acquiring a memory B-cell phenotype. It is not surprising, therefore, given the effect of latency-associated genes on cellular function, that all EBV diseases are associated with latency.

KSHV differs from EBV, as lytic replication is essential for the most common malignancy associated with this virus, i.e., KS. The lytic cycle may be activated by cellular stimuli, such as hypoxia (24), secretion of inflammatory factors (25) or co-infection with other viruses, such as human cytomegalovirus or HIV $(26,27)$. Lytic replication results in the shedding of infectious virions from the B-cell reservoir and subsequent infection of endothelial cells, where KS tumours occur (28). Although the origin of these cells (blood or lymphatic endothelium) remains to date undetermined (29), it is a hallmark of KS tumours that these cells adopt a spindle cell morphology. The majority of tumour cells remain latently infected, but low levels of virus sporadically enter the lytic cycle, meaning that there is always a small percentage of cells undergoing lytic infection $(30,31)$. It is hypothesized that lytic replication is essential for tumourigenesis, as KSHV latency is not sufficient to maintain the pool of infected endothelial cells. Furthermore, replicating KSHV expresses several oncogenic, angiogenic and antiapoptotic gene products that contribute to cancer formation (32-37), including the secretion of tumour-promoting factors, such as interleukin (IL)-6 and IL-8 (38-40).

Other malignancies associated with KSHV include the rare B-cell lymphomas primary effusion lymphoma (PEL) and multicentric Castleman's disease. Importantly, PEL cells, unlike KS-derived cells, are able to maintain high copy numbers of the latent KSHV genome when cultured and,
Table I. The human $\gamma$-herpesviruses and their associated diseases.

\begin{tabular}{lll}
\hline Virus & \multicolumn{1}{c}{$\begin{array}{c}\text { Associated } \\
\text { malignancy }\end{array}$} & \multicolumn{1}{c}{ Name } \\
\hline EBV & B-cell lymphoma & $\begin{array}{l}\text { Burkitt's lymphoma } \\
\text { Hodgkin's lymphoma } \\
\text { PTLD }\end{array}$ \\
& & $\begin{array}{l}\text { Extranodal natural killer/ } \\
\text { T-cell lymphoma }\end{array}$ \\
& T-cell lymphoma & NPC \\
& Carcinoma & Gastric carcinoma \\
& & KS \\
KSHV & Sarcoma & MCD \\
& B-cell lymphoma & PEL \\
& &
\end{tabular}

EBV, Epstein-Barr virus; KSHV, Kaposi's sarcoma-associated herpesvirus; PTLD, post-transplant lymphoproliferative disease; NPC, nasopharyngeal carcinoma; KS, Kaposi's sarcoma; MCD, multicentric Castleman's disease; PEL, primary effusion lymphoma.

therefore, provide an essential research tool for studying KSHV in vitro (41).

The gene expression profile of KSHV in healthy, immunocompetent individuals is currently unknown, as all our knowledge is gleaned from studying cancer cell lines or histological examination of malignant tissues. However, it is known that KSHV also expresses a restricted gene expression profile during latency, which is essential for the viability of these cancer cells. The expressed genes include the latency-associated nuclear antigen (LANA)1 (42), the cyclin homologue v-cyclin, the viral homologue of the cellular FLICE-inhibitory protein vFLIP and kaposin $(43,44)$. Of note, expression of vFLIP alone is sufficient to alter the morphology of cultured endothelial cells to the characteristic spindle cells of KS $(45,46)$.

There is much yet to learn about EBV and KSHV; these two viruses have been extensively investigated in recent years, particularly using proteomic-based methods. These technologies have proved to be powerful tools in aiding our understanding of these important pathogens and may help elucidate new aspects of viral biology as they become more widely used.

\section{Approaches to proteomics}

Gel electrophoresis-based methods. The first attempts to analyse the proteome occurred in the early 1970s, making use of a new technique known as polyacrylamide gel electrophoresis (PAGE) (47). Since then, there have been a number of developments in proteomic techniques and a significant increase in their utility. In this review, we provide a brief overview of the most commonly used proteomic approaches.

Gel-based methods belong to the most established techniques in proteomics and remain the preferred choice for separation and semi-quantitative identification of various 
proteins. The most commonly used method is one-dimensional (1D)-PAGE, which may be used to separate denatured protein samples according to their molecular weight in a polyacrylamide gel (47). Although this technique is used for western blot analyses in numerous laboratories, it has limitations with regards to identification of single proteins within large protein mixtures. Proteins of the same mass are detected at the same position in the gel, leading to a mixed signal.

This problem may be overcome by using two-dimensional (2D)-PAGE. With this method, the proteins are separated by their isoelectric point along a $\mathrm{pH}$ gradient in the first dimension, a process termed isoelectric focusing (48). This is followed by separation according to molecular mass. Due to the introduction of the second dimension, separation of $\leq 10,000$ proteins is possible (49). Furthermore, recent developments in protein staining techniques, such as the introduction of fluorescent dyes, have allowed not only a sensitivity of detection in the femtomolar range, but also widened the linear dynamic range by approximately three orders of magnitude (50-55). However, direct comparison between different protein samples has always been a weak point, as separate gels must be run for each sample and methodical errors may intervene with the obtained results. This has been overcome by introducing fluorescence 2D difference gel electrophoresis (2D-DIGE), which allows comparison of $\leq 3$ protein samples in one gel $(56,57)$. In this approach, the respective protein mixes are labelled with cyanine dyes (CyDyes), which bind covalently to the e-amino group of the lysine residues of the proteins. Currently, three different CyDyes are available, namely, Cy2, Cy3 and Cy5, which may be separately detected (57). Specific software, e.g., DeCyder, has been developed to help analyse the gel images. Although this technique is highly sensitive, its power is limited due to potential inconsistent labelling of proteins (56). Furthermore, 2D-DIGE does not overcome the limitations of 2D-PAGE, as all the proteins must be in a molecular weight range between 5 and $150 \mathrm{kDa}$ and a $\mathrm{pH}$ range of 3.5-10 in order to be detected $(56,58)$. Proteins that are successfully separated by 2D- or 1D-PAGE, may be further defined by mass spectrometry (MS).

MS-based methods. Over the last three decades, developments in liquid chromatography (LC), MS and protein labelling, e.g., the introduction of electrospray ionisation (ESI) and stable isotope labelling by amino acids in cell culture (SILAC), have enabled the establishment of shotgun proteomics. MS is currently commonly used for accurate peptide identification and quantification. Quantitative proteomics strategies rely on relative quantification, comparing the levels of individual peptides identified from one or more experimental samples to those levels from an untreated control sample. This provides an invaluable and powerful tool for global analysis of protein composition; however, it is important to highlight that the proteomic coverage of the genome remains a major technical challenge, as only $\sim 10 \%$ of the proteins present in the sample are identified and even fewer are quantified (59). MS-based proteomic approaches may be used to identify and quantify proteins from a whole-cell lysate, an isolated organelle/region of a cell population (e.g., nuclear envelope), or a particular tissue or organism. As previously alluded to, further protein purification may be achieved by means of LC or gel electrophoresis.
These strategies allow the removal of high-abundance proteins, which would otherwise mask low-abundance proteins, while also reducing sample complexity for increased peptide identification and quantification. Following purification, equal amounts of protein from each of the samples are proteolytically digested into peptides, often using trypsin. The digested peptides are separated and ionized by LC coupled to ESI and are then subjected to tandem MS (MS/MS). The fractions undergo a first round of MS (MS1), in which the mass-to-charge ratio $(\mathrm{m} / \mathrm{z})$ of all ionised peptides is measured, generating a precursor ion spectrum. The relative intensity of each spectrum peak correlates proportionally to peptide concentration, allowing relative quantification across different experimental conditions. Individual peptide populations are then selected for collision-induced dissociation (CID) with an inert gas and a second round of MS (MS2) determines the $\mathrm{m} / \mathrm{z}$ of the peptide fragments generating a CID spectrum. This spectrum is then compared with a theoretical database produced via an in silico digest for peptide identification (Fig. 1) $(60,61)$. The majority of global proteomic analyses across different experimental conditions are currently performed using stable isotope labelling, mass tag labelling or label-free approaches (62).

SILAC. SILAC, as first described by Ong et al (63), is an in vivo metabolic labelling technique, enabling the incorporation of different atomic isotopes into proteins as they are being synthesised in the living cell. SILAC is routinely performed using cell culture systems, but it has also been applied to simple organisms (e.g., C. elegans or Drosophila melanogaster) (64) and even mice (65). Stable (non-radioactive) naturally occurring isotopes are variants of a specific chemical element (commonly $\mathrm{H}, \mathrm{C}, \mathrm{N}$ and $\mathrm{O}$ ), which contain the same number of protons but different numbers of neutrons in each atomic nucleus. Isotopes have identical chemical properties, reducing the risk of introducing experimental bias between differently labelled samples, but the isotopes have different masses; therefore, they are easily identified by MS. The cells are cultured in growth medium with specifically labelled amino acids containing either a light isotope (e.g., ${ }^{12} \mathrm{C}$-lysine and/or ${ }^{12} \mathrm{C}$-arginine) or a heavy isotope (e.g., ${ }^{13} \mathrm{C}$-lysine and/or ${ }^{13} \mathrm{C}$-arginine). ${ }^{13} \mathrm{C}$ has an extra neutron compared to ${ }^{12} \mathrm{C}$, which gives ${ }^{13} \mathrm{C}$ an increment in its mass number of $1 \mathrm{Da}$. The substitution of light ${ }^{12} \mathrm{C}$-arginine with heavy ${ }^{13} \mathrm{C}$-arginine results in a 6-Da increment in the mass spectrum of a peptide containing one arginine, as a single arginine residue contains six carbons. Trypsin cleaves lysine and arginine residues at the $\mathrm{C}$-terminus; therefore, using these particular labelled amino acids ensures that all tryptic peptides (except for the C-terminal peptide) contain at least one labelled residue. For SILAC to work effectively, the cells must be grown in labelled growth medium for a number of passages in order to incorporate isotopes into the majority of the proteins. A very high rate of isotopic incorporation (>90\%) is achieved in cells after 6-8 doublings (63). At this point, cells that have been differentially labelled undergo the desired treatment, for example, a viral infection. SILAC allows for a maximum of three different samples in a single mass spec run with the use of light (e.g., ${ }^{12} \mathrm{C}$-arginine and ${ }^{12} \mathrm{C}$-lysine; R0K0), medium (e.g., ${ }^{13}$ C-lysine and 2D lysine; R6K4) and heavy (e.g., ${ }^{13} \mathrm{C}{ }^{15} \mathrm{~N}$-arginine and ${ }^{13} \mathrm{C}{ }^{15} \mathrm{~N}$ lysine; $\mathrm{R} 10 \mathrm{~K} 8$ ) isotope-containing media. A major advantage of SILAC is that, once generated, 


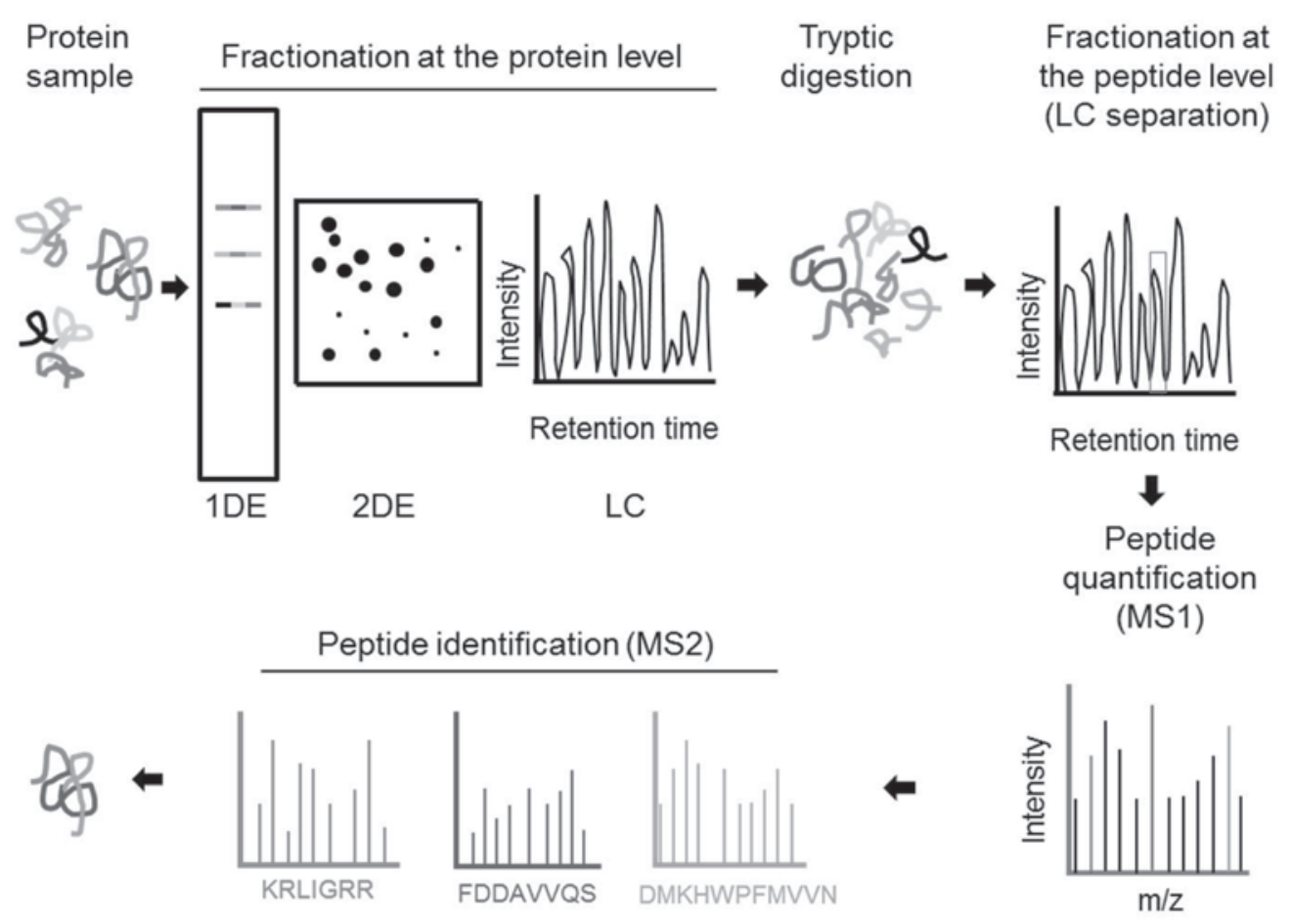

Figure 1. Proteomic analysis by mass spectrometry (MS). Protein samples are frequently separated by one- and two-dimensional electrophoresis (1DE and $2 \mathrm{DE}$, respectively) to reduce sample complexity, prior to being subjected to liquid chromatography (LC) for further separation. The samples are then loaded onto the mass spectrometer for 2 rounds of detection and the output signal is used to quantify proteins in the sample.

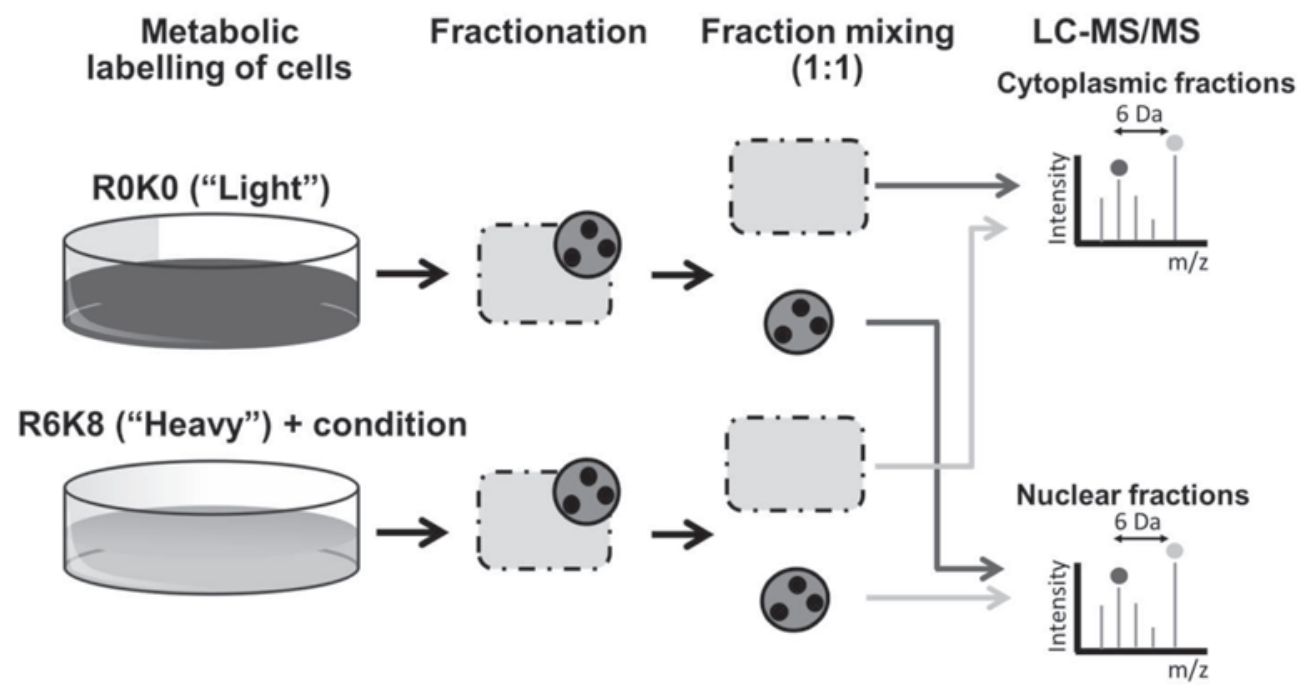

Figure 2. Combining stable isotope labelling by amino acids in cell culture (SILAC) with subcellular fractionation. Cells are grown in SILAC media for 6-8 doublings and are then fractionated into cytoplasmic and nuclear fractions. These fractions are then mixed 1:1 and sent for mass spectrometry (MS) analysis. This reduces sample complexity and minimizes the possibility of handling error. LC, liquid chromatography; $\mathrm{m} / \mathrm{z}$, mass-to-charge ratio.

labelled and unlabelled samples are mixed in equal amounts into one pooled sample. This allows all the samples to be processed together, reducing the risk of external experimental error. Furthermore, due to its accuracy, SILAC is particularly suitable for revealing relatively small changes in protein levels or post-translational modifications. A limitation of SILAC, however, is that certain cell lines may convert excess arginine into proline and, therefore, titration of arginine into the medium may be required (66). In addition, SILAC may not be applicable to cells that are sensitive to changes in the medium composition. Combining SILAC with subcellular fractionation may further reduce sample complexity (Fig. 2).

Isotope-coded affinity tagging (ICAT), isobaric tags for relative and absolute quantification (iTRAQ) and tandem mass tagging (TMT). ICAT was the first method involving the addition of isotopic tags to proteins for quantitative MS. This method was reported in 1999 by Gygi et al (67) and allows labelling of proteins present in any protein sample, including extracts from tissues or body fluids. As opposed to SILAC, 

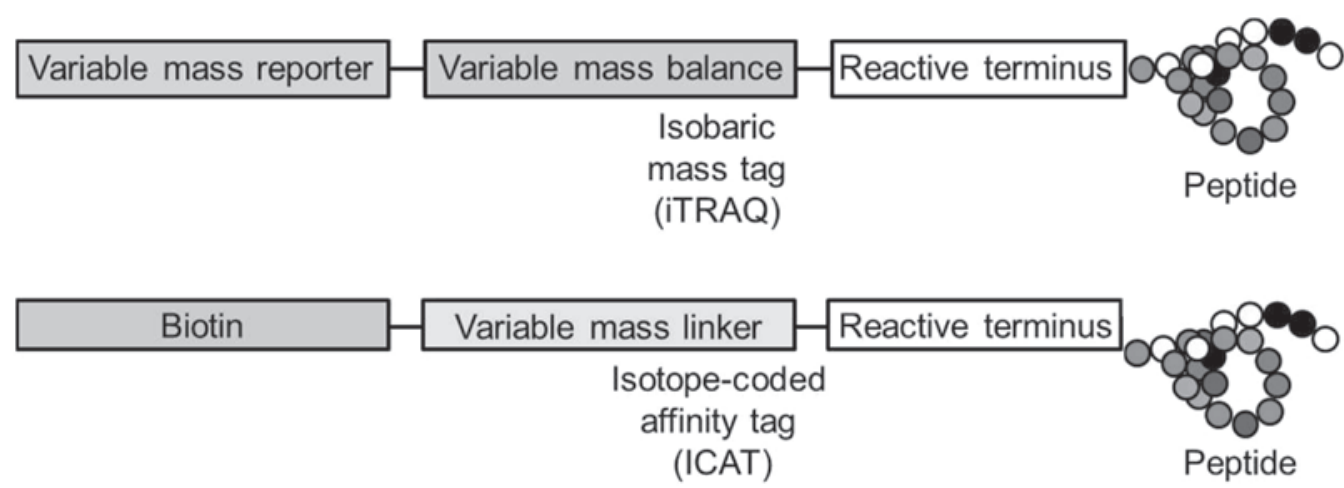

Figure 3. Isobaric mass tags and isotope-coded affinity tags. Isobaric mass tags contain a variable mass reporter for identification of sample, and are balanced to equal weights, resulting in identical behavior during the liquid chromatography separation stage. Isotope-coded affinity tags also contain a variable mass reporter (known as a linker) for sample identification and contain a biotin molecule to allow avidin affinity purification of tagged peptides. Both tags are joined to specific amino acid residues in tagged peptides through a reactive terminus.

labelling occurs in vitro following cell lysis. ICAT relies on the use of a reagent composed of three elements: a biotin molecule to enable purification of the labelled peptides with the use of avidin or streptavidin affinity chromatography; a linker group, which contains stable isotopes (light or heavy); and a reactive iodoacetyl group, which specifically reacts with free thiols on cysteine residues. Following labelling, light and heavy samples are combined into one sample, which is subjected to proteolytic digestion, affinity purification and LC-MS/MS. As labelling only occurs in proteins containing cysteine, which is a rare amino acid, ICAT inherently implies a considerable bias against proteins that lack or contain few cysteine residues; however, for the same reason, this technique is also advantageous when one wishes to reduce complexity in the protein mixture. A modification of ICAT has been devised to overcome this limitation, known as isotope-coded protein labelling (68), in which all free amino groups on intact proteins are isotopically labelled with either light or heavy tags, resulting in a higher proteomic coverage compared to ICAT.

Further techniques using tags of the same mass have also been developed for use in MS-based quantitative proteomics, most commonly known as iTRAQ and TMT. These techniques enable examination of four, six or even eight different experimental conditions in a single MS experiment with the use of up to eight different isobaric mass tags. These tags are all of the same mass and consist of three components: an $\mathrm{N}$-hydroxysuccinimide ester group, which reacts with primary amino groups; a mass tag (reporter) group; and a balance (carbonyl) group. The reporter group is based on $\mathrm{N}$-methylpiperazine and contains a unique number of heavy ${ }^{13} \mathrm{C}$ and ${ }^{15} \mathrm{~N}$ substitutions. The masses added by each tag are compensated with balance groups of variable masses to make all tags equal in mass, meaning that the same protein from different samples will behave in the same manner during LC and MS1. Each experimental condition is enzymatically digested individually, the peptides are then tagged and the samples are pooled for LC-MS/MS analysis. Upon collision-induced dissociation, the mass tags are cleaved from the balance groups. During MS2, the intensity of the different tags are used for relative peptide quantification, whereas the peptide fragment ions are sequenced to determine protein identity. As isotopic labelling occurs after enzymatic digestion, the sample complexity is increased and more abundant proteins are overrepresented during MS identification (69). iTRAQ and TMT may also be used for absolute quantification, in which the exact amount of a protein can be measured. The differences between these isobaric mass tags and the previously mentioned isotopecoded affinity tags are shown in Fig. 3.

Label-free MS analysis. As the labelling of proteins is always limited by various factors, two label-free techniques have recently been developed for use in quantitative proteomics.

Spectral counting is simply based on counting mass spectra collected during a tandem MS run, as previous studies demonstrated that the number of these mass spectra is proportional to the concentration of a given peptide and, therefore, its respective protein in the sample $(58,70)$. Prior to ionisation and MS/MS, the peptides resulting from the initial protein trypsinisation are separated by LC. Elution of highly concentrated peptides into the MS/MS ionisation chamber occurs over a longer period compared to the elution of peptides with lower concentrations; therefore, more spectra of the more concentrated peptides may be acquired compared to the less concentrated peptides (70). Although modern LC systems are capable of very accurate separation, there is always the possibility of co-eluting peptides when analysing complex protein samples (58). This has resulted in critical discussion regarding this label-free method, particularly as no other physicochemical properties of the individual peptides are measured (71). However, there have been recent developments to improve spectral counting and moving from relative quantification to absolute protein measurement. The normalised spectral abundance factor (NSAF), the distributed NSAF and the normalised spectral index take into account not only the number of spectra, but also peptide count, protein length, sample-to-sample variation for replicate analyses, fragment-ion intensity and the distribution of spectral counts of peptides shared by different proteins (72-74). The absolute protein expression aims for absolute protein measurement by calculating the proportionality of expected peptides after trypsinisation and the observed peptides for a given protein after MS/MS (75).

Measurement of chromatographic peak areas (or MS signal intensities) is the second method used for label-free quantitative proteomics. The peptides are separated by LC according to their 


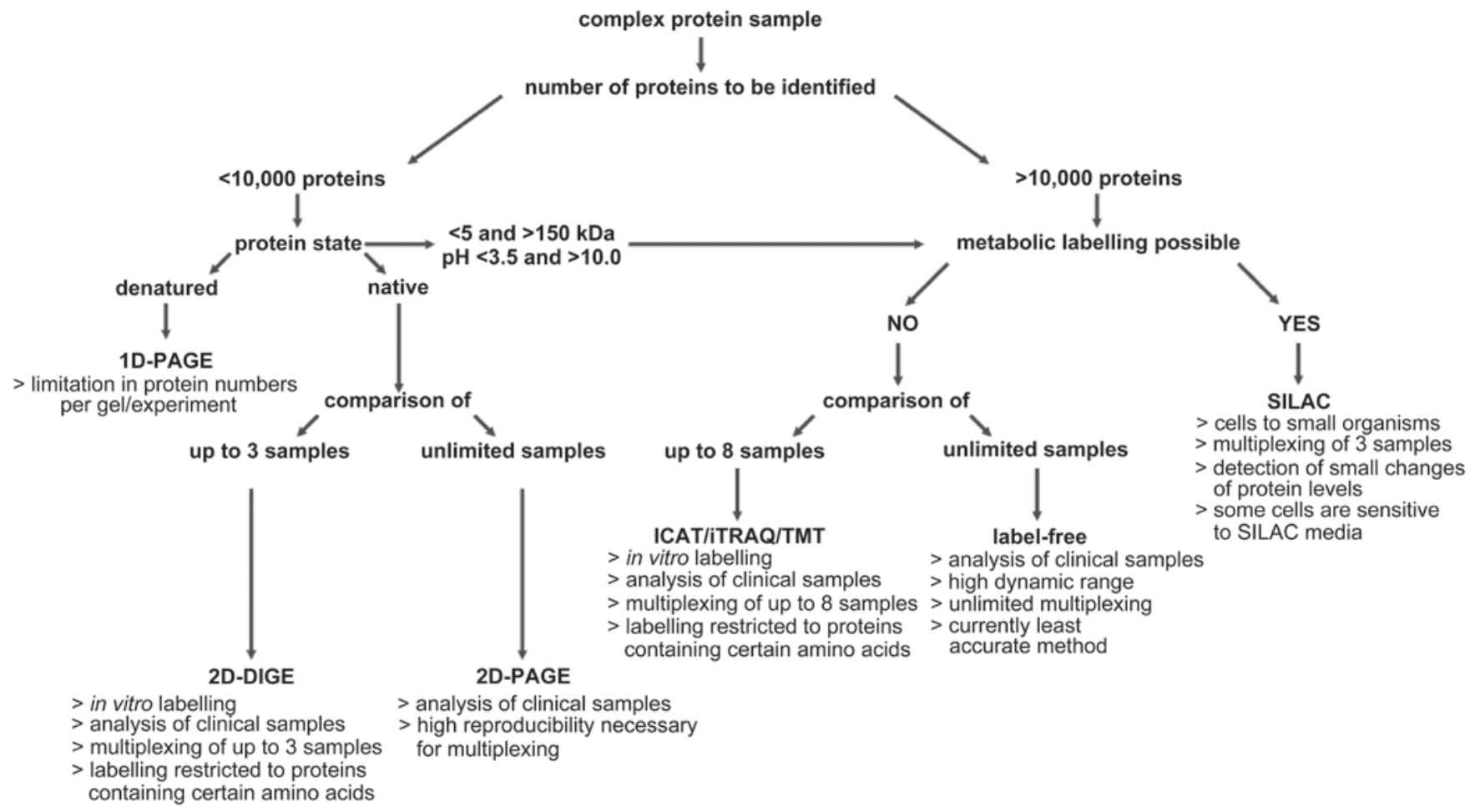

Figure 4. Several approaches to proteomic studies. This flowchart suggests which proteomic technique to use based on the properties of the sample being analysed. 1D, one-dimensional; 2D, two-dimensional; PAGE, polyacrylamide gel electrophoresis; SILAC, stable isotope labelling by amino acids in cell culture; ICAT, isotope-coded affinity tagging; iTRAQ; isobaric tags for relative and absolute quantification; TMT, tandem mass tagging; DIGE, difference gel electrophoresis.

physical properties, e.g., charge or hydrophobicity, followed by ionisation and detection of peptide precursor ions in a mass spectrometer. Every precursor ion generates a specific monoisotopic peak, the intensity of which may be shown as function of the LC retention time in an extracted ion chromatogram. The chromatographic peak areas for each peptide, determined by the area under the curve, correlate with the abundance of the respective peptide. Therefore, a relative quantification between different protein samples is possible (76-78). However, this requires a high level of reproducibility between the different samples $(71,78)$. Moreover, due to identifying and focusing on specific precursor ions at an early stage, there may be a reduction in the peptides sequenced during MS/MS (79). To overcome this, two separate analyses, one LC-MS and one LC-MS/MS, may be performed for each sample. Nevertheless, newer and faster mass spectrometers are able to collect data for MS as well as MS/MS scans at a satisfying rate $(71,79)$.

Although label-free is currently the least accurate proteomic method, it is worth considering, as it displays distinct advantages. First, the money- and time-consuming step of introducing labels into proteins is not necessary, which also leads to a reduction in experimental stages. Furthermore, although multiplexing is not available for label-free samples, there is no limit for parallel experiments if all the stages of sample preparation and analyses are reproducible. Finally, label-free quantifications exhibit a higher dynamic range compared to stable isotope labelling. The merits of spectral counting, in particular, have been demonstrated when seeking large changes in protein levels compared to SILAC-labelled samples $(70,71,80)$.
There are several factors to be considered when selecting a particular method (Fig. 4), but often different approaches yield comparable results; therefore, most consideration should potentially be given to the ease of application and how well equipped a lab is to attempt any given approach.

Proteomic methodologies have been demonstrated to be useful in a wide range of biological investigations, including numerous studies of interactions between virus and host proteomes. There are a number of examples of $\gamma$-herpesvirus studies that have utilised these approaches, which have helped elucidate several aspects of their biology.

\section{Understanding $\gamma$-herpesviruses through proteomics}

EBV is an extremely successful oncovirus, infecting a predicted $90 \%$ of the population; however, very few infected individuals actually develop neoplasia. This demonstrates the ability of EBV to limit its oncogenic potential, despite expressing multiple proteins able to immortalise cells in vitro. It is not surprising, therefore, that various studies have demonstrated that EBV exerts a significant effect on the cellular transcriptome. Less attention has been given to the effect of EBV of the cellular proteome; however, this is an important consideration, as changes in mRNA abundance do not always translate into alterations at the protein level. Hence, a thorough investigation of the effect of EBV on the cellular proteome is warranted.

As the most recently discovered human herpesvirus, our knowledge of KSHV is behind that of other, more characterised viruses, such as EBV. However, over the last decade, several investigations into KSHV biology have been performed, 
utilising advanced proteomic-based technologies, which have resulted in significant advances in our understanding of this virus. As with EBV, there remains much to learn regarding KSHV biology and large systems biology approaches, such as proteomic-based studies, may play a major role in elucidating the intricacies of these two complicated pathogens.

Proteomic analysis of $\gamma$-herpesvirus virions. Early analyses of viral proteomes often focus on the composition of the mature viral particle, as the proteins found within are often instrumental to a successful infection. As with all herpesviruses, EBV and KSHV have complex virions, constructed of an inner nucleoprotein core, a viral capsid, an inner tegument layer and a lipid envelope (81). Surprisingly, despite being the first true human oncovirus to be discovered, only a single study has used proteomic analyses to comprehensively study EBV virion composition (82). In that study, the EBV lytic cycle was induced from latently infected B cells by the ectopic expression of the EBV lytic switch protein, Zta. Mature virus particles were purified using density gradients, de-enveloped and subjected to in-gel trypsin digestion and LC-MS/MS. This analysis largely demonstrated that EBV virion morphogenesis follows that of other herpesviruses, such as the model herpesvirus, herpes simplex virus (HSV). ICAT was also used to determine how likely a protein was to be associated with the envelope or the EBV capsid. In this example, ${ }^{12} \mathrm{C}$ 'light' labelled enveloped virions were mixed with ${ }^{13} \mathrm{C}$ 'heavy' labelled virions that had been treated with detergent to remove the envelope. Following normalisation between samples on the major capsid protein, it was observed that several cellular proteins were major components of mature EBV virions, including $\beta$-actin (one of the most abundant tegument proteins), cofilin, tubulin, heat shock protein (Hsp)70, Hsp90 and other actin-associated proteins (ARPs), such as profilin, ezrin, radixin, moesin and $\alpha$-actinin. Hsp70, $\alpha$-tubulin, $\beta$-tubulin and actin were shown to be tightly associated with capsids, suggesting that they may be involved in stabilising the capsids or that they may be required immediately after de-envelopment during de novo infections. Additional functions for the cellular proteins may include EBV virion morphogenesis and egress.

Initial determinations of the KSHV virion components were performed in a similar manner, subjecting mature virions purified by density gradient to $1 \mathrm{D}$-gel electrophoresis, in-gel tryptic digest and MS (83). In total, 24 proteins of structural, regulatory and signalling roles were identified, including 5 capsid proteins, 8 tegument proteins, 8 glycoproteins found within the lipid envelope and a further 3 proteins associated with the virion. Of note, several of the identified proteins were of cellular origin, including a number of proteins also found in EBV virions, such as $\beta$-actin and the ARPs ezrin and moesin. The roles of these proteins in the viral particle have not yet been elucidated; however, their presence suggests that they are required for virion stability or during the early stages of infection.

The chaperones Hsp90 and Hsc70, identified in the virions of a wide range of viral families, were also identified in the tegument of KSHV. These proteins have been shown to play numerous roles in viral processes, ranging from relocalization of key viral factors to aiding in the reverse transcription of retroviral genomes. Furthermore, the KSHV lytic reactivator protein replication and transcription activator (RTA) (84) was identified as a component of the KSHV tegument, suggesting that RTA drives a round of lytic gene expression immediately after infection. These studies demonstrated the efficacy of using reduced-complexity samples with MS-based proteomics to identify a subset of viral and cellular proteins involved in different aspects of viral biology.

Elucidating the effects of fully competent $\gamma$-herpesvirus infection through proteomics. In an attempt to gain understanding of EBV pathogenesis, Alsayed et al (85) performed a proteomic analysis of PTLD tissues from solid organ transplant patients using a protein microarray technique. The proteins that were dysregulated included proteins of the phosphoinositide 3-kinase (PI3K)/mammalian target of rapamycin, nuclear factor- $\kappa \mathrm{B}$ and $\mathrm{Hsp} 90$ pathways, all of which are entirely consistent with the latency III gene expression profile in PTLD. Interestingly, when specific inhibitors of these proteins were then tested in $\mathrm{EBV}^{+}$and $\mathrm{EBV}^{-}$cell lines, they were found to be specifically cytotoxic in the $\mathrm{EBV}^{+}$lines. Indeed, it was recently demonstrated that the Hsp90 inhibitors geldanamycin, 17-allylamino-17-demethoxygeldanamycin and 17-dimethylaminoethylamino-17-demethoxygeldanamycin specifically decreased EBNA1 translation, leading to cytotoxicity of $\mathrm{EBV}^{+}$cells and significantly inhibiting lymphoproliferative disease in severe combined immunodeficiency mice $(86,87)$. Together, these early results highlighted the value of proteomics in identifying novel therapeutic targets.

Proteomic analyses have also been applied in order to understand how the human $\gamma$-herpesviruses manipulate intercellular communication. In addition to the secretion of soluble factors that promote the establishment of latency (or indeed, tumourigenesis), virally infected cells also release modified exosomes. These are a class of endosomal-derived membrane microvesicles from multivesicular bodies that, after release, may fuse with recipient cells, into which they release their contents and modulate biological activities. Although this is a normal physiological process, there is evidence suggesting that altered exosome function may be pathogenic, particularly during malignancy. It was demonstrated that EBV-infected cells release exosomes containing virally-encoded miRNAs and proteins and that these factors are able to alter target cell function (88-90). For example, EBV's major oncoprotein LMP1 was highly enriched in exosomes secreted from nasopharyngeal carcinoma (NPC) cells and the transfer of these to recipient cells activated several LMP1-associated signalling cascades, including PI3K/AKT and mitogen-activated protein kinase/extracellular signal-regulated kinase (91). LMP1 has also been identified in B-cell-secreted exosomes and this may be important for inducing proliferation and B-cell survival, a crucial step in the establishment of EBV latency and colonisation.

However, there remains the major question whether viral infections dictate the composition of secreted exosomes. This was recently addressed by Meckes et al (92) using a combination of MS and 2D-DIGE to determine the repertoire of proteins in $\mathrm{EBV}^{+}, \mathrm{KSHV}^{+}$and dually infected cell line exosomes, compared to non-infected counterparts. In that study, a total of 871 proteins were identified from all exosome preparations and 449 of these proteins were common among infected and 
non-infected cell lines. Therefore, several hundred exosome proteins were enriched as a result of $\gamma$-herpesvirus infections. Some of these proteins were specific to EBV $(n=93)$ and others to KSHV $(n=22)$, suggesting that these different viruses manipulate the exosome proteome in diverse ways, possibly in order to optimally promote their particular life cycles. Furthermore, a 2D-DIGE, coupled with a spectral count-based quantitative proteomic approach, was employed to compare the exosomes of $\mathrm{LMP}^{+}$and $\mathrm{LMP}^{-}$cells, confirming that several of the alterations in the EBV-associated exosome cargo were due to LMP1. Moreover, the pathway analysis revealed that both EBV- and KSHV-associated exosome components largely affect similar cellular pathways, although KSHV exosomes were enriched in proteins involved in cellular metabolism, whereas EBV-associated exosomes were geared towards cellular signalling pathways.

The abovementioned findings demonstrate that proteomic-based research is able to identify multiple protein pathways that are altered by related viruses and put these into the context of a viral infection. However, a potential caveat that exists in a majority studies regarding the human $\gamma$-herpesviruses is the lack of isogenic virus-negative counterparts to rule out the possibility that some differences are purely cell type-specific. Despite this caveat, however, these studies enhance our understanding of how KSHV and EBV manipulate the tumour microenvironment and provide potential new avenues for therapeutic intervention. Interestingly, several of the proteins identified in mature $\gamma$-herpesvirus virions have also been detected in their exosomes (e.g., actin and ezrin), signifying the possibility that virally induced exosomes may mimic viral infection. If this is true, the extent to which it may benefit virus colonisation remains to be determined. The next challenge will be to elucidate how viruses dictate exosome proteomes and how this translates in vivo.

Investigating the interactions and effects of specific viral factors. Proteomic approaches have also been used to investigate the function of individual viral proteins. The EBNA2 protein is considered to be the master transcriptional activator, switching on the expression of all of EBV's immortalising genes, as well as various cellular genes known to be critical for EBV infection, such as CD23. In an attempt to identify proteins altered due to EBNA2 expression, 2D-PAGE was applied to a protein sample from a conditionally immortalised B-cell line, in which EBNA2 is activated by translocation to the nucleus, due to the addition of oestrogen (93). In that study, EBNA2 target proteins and the proteins that were induced during primary B-cell infection were shown to correlate, highlighting the major role of EBNA2 during the establishment of latency and B-cell immortalisation. A total of 20 differentially expressed proteins were identified, 12 of which were upregulated, including the protein synthesis and degradation factors eIF5a, P0, PA28 $\gamma$ and ubiquitin thiolesterase L3, as well as two proteins involved in nucleotide metabolism, Nm23-H1 and pyrophosphatase. Some of the best characterised EBNA2-responsive proteins were not identified, however, suggesting incomplete coverage due to the nature of the proteomic approach employed.

Similarly, the effect of individual KSHV protein expression has been investigated using a gel-based approach.
2D-DIGE was successfully utilised to analyse the protein content of endothelial cells expressing the KSHV vFLIP protein (94). This study identified 14 proteins upregulated upon vFLIP expression, the most highly upregulated of which was manganese superoxide dismutase (MnSOD). Interestingly, this is an antiapoptotic enzyme that has also been shown to be upregulated in KSHV-infected cells. Importantly, previous transcriptome analyses also identified MnSOD to be upregulated at the RNA level, demonstrating the possibility of combining proteomics and transcriptomics approaches to validate and enhance results $(94,95)$ (see Section 4).

Another proteomic-based study of EBV investigated the transformation potential of the EBV LMP1 oncoprotein in NPC cell lines (96). In that study, a total of 250 proteins were detected by iTRAQ-based analysis, 12 of which were shown to be differentially expressed (although proteins that changed as little as 1.33 -fold were also considered), including 6 proteins involved in signal transduction: voltage-dependent anion channel 1, S100-A2, hormone-induced protein-70, ubiquitin, tumour protein translationally-controlled 1-like protein and $4 \mathrm{~F} 2 \mathrm{hc}$, as well as 3 proteins pertaining to cyctoskeleton formation: keratin-75, TB8 and dynein light chain 1.

The first SILAC-based study designed to investigate a small number of changes in specific subcellular compartments utilised the expression of the KSHV K5 protein (97). Modulation of the host immune response, particularly via major histocompatibility complex (MHC) class I molecules, is a common trait amongst pathogens. The KSHV-encoded $\mathrm{K} 3$ and $\mathrm{K} 5$ proteins, which function as membrane-bound RING-CH ubiquitin ligases and target MHC I for lysosomal degradation, are homologues of the K3 family of viral immune modulators. Importantly, other viral downregulators of MHC I are also known to target additional transmembrane proteins pertaining to different cellular pathways. In order to identify any novel K5 targets, plasma membrane, endoplasmic reticulum (ER) and Golgi apparatus were isolated from labelled control cells or cells expressing K5. In addition to the expected downregulation of MHC I, 3 novel peptides were found to be downregulated in the plasma membrane of cells expressing K5: activated leukocyte cell adhesion molecule, bone marrow stromal cell antigen 2 (BST-2) and syntaxin-4. The identification of these proteins marked a precedent in SILAC proteomics, as it demonstrated a new approach for identifying additional targets of viral proteins. BST-2 (also known as tetherin) is of particular interest, as this protein was first identified as a host restriction factor that prevents the release of budding retroviruses from the plasma membrane. $\mathrm{HIV}$ is able to counteract BST-2 via its auxiliary protein, viral protein unique, by co-opting a cellular ubiquitin ligase, $\beta \operatorname{TrCP}$, resulting in BST-2 degradation. Subsequent studies confirmed that BST-2 is indeed a bona fide target of K5, although it was shown to be subjected to proteasomal degradation as it left the ER, rather than being degraded at the plasma membrane by the lysosome (as is true of other K5 targets). Therefore, similar to HIV, KSHV has also developed a mechanism of circumventing BST-2 function, although this virus has evolved its own specialised ubiquitin ligase, namely K5.

MS-based proteomics have also been used to identify cellular proteins binding to the terminal repeat regions (TRs) of the KSHV genome during latency. KSHV TRs contain the 
latent origin of viral replication and play a major role in the maintenance and spread of the viral episome among dividing cells. In order to further characterise these important regulatory elements, TR sequences were used to affinity capture proteins from KSHV-positive cell lysates, which were subjected to MS-based proteomic analysis (98). In total, 123 proteins were found to bind the TRs of KSHV and further analysis on 4 of these proteins, namely ataxia telangiectasia and Rad3-related protein, transcription activator BRG1, nucleophosmin 1 and poly(ADP-ribose) polymerase 1, demonstrated that they associate with the KSHV-encoded LANA. This suggested that proteins binding TR sequences may play a role in viral latency.

Furthermore, by employing affinity capture methods coupled to 1D-PAGE and MS, glutathione S-transferase-tagged $\mathrm{N}$ - and C-terminal regions of LANA were used to capture and identify protein interaction partners of LANA (99). Several proteins were found to bind to the N-terminus $(n=53)$ and the C-terminus $(n=56)$ and functional analysis of the protein groups identified 27 different categories, including ATPases, ribonucleoproteins and cytoskeletal proteins. Whilst the majority of these novel interactions are yet to be fully characterised, most of the proteins that bound LANA were found to be transcription factors and DNA-binding proteins, suggesting that LANA plays an important role in transcriptional control.

More recently, our laboratory has undertaken SILAC-based proteomics to elucidate the effects of the essential KSHV mRNA processing protein, ORF57 $(100,101)$, on cellular pathways. To this end, an inducible HEK293-based system expressing ORF57 was utilised. To maximise the data obtained from the study, cellular fractionation was performed to separate the major cellular compartments where ORF57 is known to be present: the cytoplasm, nucleoplasm and nucleoli (102). Predictably, as ORF57 is a known viral RNA processing protein, its expression led to an enrichment of proteins involved in RNA processing, RNA post-transcriptional modification and gene expression, particularly in the nucleolar fraction, where ORF57 is highly abundant $(103,104)$. Importantly, the analysis also identified an enrichment in DNA repair proteins that led to the discovery of a novel role for ORF57 in genome instability (105). That study highlighted the significance of large-scale proteomics studies for identifying novel proteins or cellular pathways of interest. Moreover, cellular fractionation and isolation of cellular compartments or organelles linked to SILAC proteomics will substantially increase the flexibility of this type of approach.

Combining protein immunoprecipitation with mass spec-based quantitative proteomics provides another way to reduce sample complexity and gain greater insight into the intricacies of viral infection. Malik-Soni and Frappier (106) have used this method to elucidate the interaction partners of the EBV EBNA1 protein in EBV-positive and -negative NPC cell lines. In that study, EBNA1 was found to have very similar interactomes in the two different cell backgrounds and these interaction profiles largely agreed with previous studies using HEK 293T cells. Furthermore, the EBNA1 interaction partners in AGS cells infected with latent EBV were highly similar to those in lytically infected cells, demonstrating that several of the EBNA1-host protein interactions occur independent of EBV infection. Of note, EBNA1 was also shown to associate with numerous heterogeneous nuclear ribonucleoproteins and the La protein, which associated with the $3^{\prime}$ ends of newly synthesized small RNAs and had previously been shown to bind EBV EBERs (107). The interaction between La and EBNA1 only occurred in the context of full EBV infection and was ablated by RNase treatment, suggesting this interaction is bridged by EBERs. EBNA1 also appeared to interact with the nucleolar protein B23 (nucleophosmin), a protein that frequently plays a role in infection with other viruses, such as KSHV and HIV-1 $(98,108)$.

Other large-scale studies have also added to our knowledge of the KSHV proteome, although not strictly through a proteomic approach. For example, a large study was performed by Sander et al (109) with the aim to map the subcellular localisation of every KSHV protein in HeLa cells in order to elucidate potential roles of proteins within the context of the viral infection with regard to their location.

Greater understanding of a viral proteome may also be used to create novel diagnostic tools. Recently, a 96-well format multiplex bead-based assay was used to analyse the serological response of 2 groups of individuals previously diagnosed with KSHV-associated malignancies $(n=36)$ or with a low likelihood of KSHV infection $(n=43)$ to 73 different recombinant proteins encompassing most of the KSHV proteome (110). Historically, the KSHV ORF73 and K8.1 proteins have been used to determine whether an individual is infected; however, the patterns of reactivity in those infected with KSHV were found to be highly variable. That study identified 6 more viral proteins that may be tested for to distinguish between KSHV-positive and -negative individuals, providing a more powerful and reliable tool for clinical KSHV serodiagnosis.

This selection of proteomic-based research demonstrates the power of the numerous techniques that have been developed in this field. Combining proteomic techniques with other large-scale systems biology approaches may provide an even greater insight into the various processes occurring within populations.

\section{Combining OMICS approaches to maximise function}

It is becoming increasingly apparent that utilising multiple high-throughput systems techniques can create a synergy, enabling greater insight into more biological processes, rather than by relying on an individual technique. As the field of large-scale proteomics is still in its infancy, it is likely that the full potential of combining OMICS approaches has not yet been realised, although there are numerous published examples of groups combining two or more methods to answer newly arising questions.

Currently, the majority of proteomic-based studies focus on model organisms, for which there exists a database of encoded proteins. A recently developed approach, known as Proteomics Informed by Transcriptomics (PIT), combines deep sequencing-based transcriptomics (RNA-seq) with MS-based quantitative proteomics (111). PIT allows the interpretation of the proteomes of multiple species in the same sample, or of species without a complete reference genome, using the transcriptome data to reveal any proteins that may be present. This approach may also be used to identify changes in mRNA levels that are not reflected at the protein level, allowing the identification of potential post-transcriptional degradation targets. Furthermore, combining these different OMICS procedures 
allows researchers to overcome the limitations of an individual technique, such as incomplete coverage of the transciptome, due to high mRNA turnover rate, or of the proteome, due to the issues previously mentioned with LC-MS/MS analysis.

Other attempts to apply large-scale OMICS techniques to non-model species rely on the sequencing and annotation of a novel reference genome. The initial interpretation of a novel genome is often heavily guided by using sequence homology to closely related species and in silico predictions of open reading frames. This commonly results in incomplete genome annotations, often ignoring non-canonical elements, such as transcription start sites or genomic regions that lack sequence homology to other previously characterised genes. Indeed, some of the most interesting aspects of $\mathrm{KSHV}$ and EBV biology involve EBERs, EBNA1 and KSHV LANA, all of which could be missed using these traditional methods of genome annotation. However, the combination of LC-MS/MS-based proteomics with custom tiling arrays has filled in a number of gaps in the viral genome and identified several novel genes (112). That previous study demonstrated that combining two or more high-throughput approaches may compensate for the limitations of each individual approach and further demonstrated that combining transcriptome and proteome data may also provide some insight into the stability of the detected transcripts and proteins.

Another recently identified gap in the viral genome annotations came in the form of small non-coding RNAs, named miRNAs, which are primarily involved in the repression of translation and have been hailed as one of the most important breakthroughs in biology $(113,114)$. The identification and characterisation of functional miRNAs has proved to be a difficult endeavour in recent years, with numerous predictive algorithms being designed to elucidate target mRNA molecules. A number of these algorithms, however, rely on assumptions about miRNAs binding to target sequences at the 3 ' end of the target mRNAs, despite other mechanisms having been demonstrated (115). Even when this simplistic approach is applied, it often results in a high rate of false-positives and predicts a large list of potential targets that could not feasibly be experimentally validated. Using deep sequencing, aberrantly expressed miRNAs have been identified in osteopetrosis patients and proposed targets were identified by their altered protein expression using iTRAQ proteomics (116). This approach led to the identification of 96 novel miRNAs in the osteopetrosis samples and also provided a useful method for identifying miRNA-target pairs with greater confidence compared to that which could be achieved by relying on predictive algorithms alone. A similar approach has also been used to identify targets of several viral miRNAs encoded by KSHV, whereby pulsed SILAC was employed to identify host cell genes repressed at the protein level upon expression of individual viral miRNAs (117). While it may be possible to identify some miRNA-mRNA target pairs using deep sequencing alone, this may result in only identifying degraded mRNA targets and not those for which translation is inhibited by other means (118).

Until recently, $32-39 \%$ of the predicted genes on chromosome 1 alone lacked any protein identification according to the Chinese Human Chromosome Proteome Consortium (119), despite the publishing of a complete draft of the human genome over 10 years ago. In a recent effort to characterize all the missing proteins, an integrative OMICS approach was employed, combining MS-based proteomics, RNAseq and ribosome profiling, an emerging technique that identifies the translation potential of mRNAs present in a sample (120). This approach, utilising proteome, transcriptome and translatome data, reduced the as yet uncharacterised genes to $\sim 15 \%$, most of which are predicted to be tissue-specific.

\section{Future perspectives}

Single-cell sequencing is now a reality and numerous studies are currently being undertaken $(121,122)$. This technology provides greater insight into the biology of heterogeneous populations of cells, e.g., a tumour, in which many different cell states contribute to the overall function. Similarly, single-cell proteomic methods are currently being devised, utilising an antibody-bound chip, analogous to microarray technology (123). These approaches are expected to take high-throughput systems biology approaches to new heights and will also generate significantly larger datasets, for which new strategies will be required.

Our ability to interpret the data produced via high-throughput experimental technologies is continuously improving. There are currently numerous online tools widely available for use, such as PepTracker (124), DAVID (125) and STRING (126), that allow scientists with minimal understanding of bioinformatics to obtain useful data from large proteomic datasets. Many of these systems biology-based web applications are available free of charge to the academic community. The aim of the Proteomics Specification in Time and Space project is to keep improving the utility of large datasets through the development of analysis tools and the application of new methodologies (127).

The increasing trend towards utilising large-scale systems biology across the scientific community has led to an increasing availability of OMICS datasets. Indeed, multiple projects have been established in order to set up and maintain large data repositories, such as the ProteomeXchange and RNA-seq Atlas web applications, allowing the wider scientific community to use data previously obtained by other groups to inform their own research $(128,129)$. This is highlighted by a recent study using 42 previously published and 7 unpublished Drosophila phenotypes inferred by RNAi screens (130). This approach enabled a 'signed' protein-protein interaction network to be constructed, consisting of 6,125 interactions between 3,352 proteins. Subsequent testing of the network revealed a high predictive power. As more OMICS datasets become freely available, the power of such predictive models will increase, as will our collective understanding of the questions we can answer by using them. It is important to remain critical, however, and not rely solely on computational prediction biology. Despite the success of many of these projects, the data require validation in a wet lab environment.

Regarding EBV and KSHV, further research using large-scale systems biology approaches will help develop our understanding of the intricacies of infection. Proteomic screens are currently routinely performed and it will be of particular interest to view the results of OMICS-based analysis of numerous single cells infected with fully competent $\gamma$-herpesviruses. The challenges arising from 
this will be validating these studies and attempting to link them to clinically relevant applications for these important pathogens.

\section{Acknowledgements}

The authors would like to thank all the members of the Whitehouse Laboratory for the useful discussions. This study was supported in part by BBSRC, YCR, Wellcome Trust and AICR.

\section{References}

1. Epstein MA, Achong BG and Barr YM: Virus particles in cultured lymphoblasts from Burkitt's lymphoma. Lancet 1: 702-703, 1964

2. Chang Y, Cesarman E, Pessin MS, Lee F, Culpepper J, Knowles DM and Moore PS: Identification of herpesvirus-like DNA sequences in AIDS-associated Kaposi's sarcoma. Science 266: 1865-1869, 1994.

3. Henle G, Henle W, Clifford P, et al: Antibodies to EpsteinBarr virus in Burkitt's lymphoma and control groups. J Nat Cancer Inst 43: 1147-1157, 1969.

4. Kieff E and Rickinson AB: Fields' Virology. Knipe DM and Howley PM (eds). Vol 2. 6th Edition. Lippincott Williams and Wilkins, Philadelphia, pp2655-2700, 2007.

5. Damania B and Pipas JM (eds): DNA Tumour Viruses. 1st Edition. Springer, New York, NY, pp205-216, 2009.

6. Taylor GS and Blackbourn DJ: Infectious agents in human cancers: lessons in immunity and immunomodulation from gammaherpesviruses EBV and KSHV. Cancer Lett 305: 263-278, 2011.

7. Preiser W, Szép NI, Lang D, Doerr HW and Rabenau HF: Kaposi's sarcoma-associated herpesvirus seroprevalence in selected German patients: evaluation by different test systems. Med Microbiol Immun 190: 121-127, 2001.

8. Simpson GR, Schulz TF, Whitby D, et al: Prevalence of Kaposi's sarcoma associated herpesvirus infection measured by antibodies to recombinant capsid protein and latent immunofluorescence antigen. Lancet 348: 1133-1138, 1996.

9. Engels EA, Sinclair MD, Biggar RJ, Whitby D, Ebbesen P, Goedert JJ and Gastwirth JL: Latent class analysis of human herpesvirus 8 assay performance and infection prevalence in sub-saharan Africa and Malta. Int J Cancer 88: 1003-1008, 2000

10. Mesri EA, Cesarman E and Boshoff C: Kaposi's sarcoma and its associated herpesvirus. Nat Rev Cancer 10: 707-719, 2010.

11. Parkin DM, Sitas F, Chirenje M, Stein L, Abratt R and Wabinga H: Part I: Cancer in indigenous Africans - burden, distribution, and trends. Lancet Oncol 9: 683-692, 2008.

12. Ambroziak JA, Blackbourn DJ, Herndier BG, et al: Herpes-like sequences in HIV-infected and uninfected Kaposi's sarcoma patients. Science 268: 582-583, 1995.

13. Kurth J, Spieker T, Wustrow J, Strickler GJ, Hansmann LM, Rajewsky K and Küppers R: EBV-infected B cells in infectious mononucleosis: viral strategies for spreading in the B cell compartment and establishing latency. Immunity 13: 485-495, 2000.

14. Webster-Cyriaque J, Duus K, Cooper C and Duncan M: Oral EBV and KSHV infection in HIV. Adv Dent Res 19: 91-95, 2006

15. Tugizov SM, Berline JW and Palefsky JM: Epstein-Barr virus infection of polarized tongue and nasopharyngeal epithelial cells. Nat Med 9: 307-314, 2003.

16. Blackbourn DJ, Lennette ET, Ambroziak J, Mourich DV and Levy JA: Human herpesvirus 8 detection in nasal secretions and saliva. J Infect Dis 177: 213-216, 1998.

17. Pauk J, Huang ML, Brodie SJ, et al: Mucosal shedding of human herpesvirus 8 in men. New Engl J Med 343: 1369-1377, 2000.

18. Goodwin DJ, Walters MS, Smith PG, Thurau M, Fickenscher H and Whitehouse A: Herpesvirus Saimiri open reading frame 50 (Rta) protein reactivates the lytic replication cycle in a persistently infected A549 cell line. J Virol 75: 4008-4013, 2001.

19. Cesarman E: Gammaherpesvirus and lymphoproliferative disorders in immunocompromised patients. Cancer Lett 305: 163-174, 2011.

20. Klein E, Kis LL and Klein G: Epstein-Barr virus infection in humans: from harmless to life endangering virus-lymphocyte interactions. Oncogene 26: 1297-1305, 2007.
21. Speck SH and Ganem D: Viral latency and its regulation: lessons from the gamma-herpesviruses. Cell Host Microbe 8: 100-115, 2010.

22. Babcock GJ, Decker LL, Volk M and Thorley-Lawson DA: EBV persistence in memory B cells in vivo. Immunity 9: 395-404, 1998

23. Babcock GJ, Hochberg D and Thorley-Lawson AD: The expression pattern of Epstein-Barr virus latent genes in vivo is dependent upon the differentiation stage of the infected B cell. Immunity 13: 497-506, 2000.

24. Davis DA, Rinderknecht AS, Zoeteweij JP, et al: Hypoxia induces lytic replication of Kaposi sarcoma-associated herpesvirus. Blood 97: 3244-3250, 2001.

25. Blackbourn DJ, Fujimura S, Kutzkey T and Levy JA: Induction of human herpesvirus- 8 gene expression by recombinant interferon gamma. AIDS 14: 98-99, 2000.

26. Vieira J, O'Hearn P, Kimball L, Chandran B and Corey L: Activation of Kaposi's sarcoma-associated herpesvirus (human herpesvirus 8) lytic replication by human cytomegalovirus. J Virol 75: 1378-1386, 2001

27. Zeng Y, Zhang X, Huang Z, et al: Intracellular Tat of human immunodeficiency virus type 1 activates lytic cycle replication of Kaposi's sarcoma-associated herpesvirus: role of JAK/STAT signaling. J Virol 81: 2401-2417, 2007.

28. Wilson SJ, Tsao EH, Webb BL, et al: X box binding protein XBP-1s transactivates the Kaposi's sarcoma-associated herpesvirus (KSHV) ORF50 promoter, linking plasma cell differentiation to KSHV reactivation from latency. J Virol 81: 13578-13586, 2007.

29. Roth WK, Brandstetter H and Sturzl M: Cellular and molecular features of HIV-associated Kaposi's sarcoma. AIDS 6: 895-913, 1992.

30. Staskus KA, Zhong W, Gebhard K, et al: Kaposi's sarcoma-associated herpesvirus gene expression in endothelial (spindle) tumor cells. J Virol 71: 715-719, 1997.

31. Orenstein JM, Alkan S, Blauvelt A, Jeang KT, Weinstein MD, Ganem D and Herndier B: Visualization of human herpesvirus type 8 in Kaposi's sarcoma by light and transmission electron microscopy. AIDS 11: F35-F45, 1997.

32. Arvanitakis L, Geras-Raaka E, Varma A, Gershengorn MC and Cesarman E: Human herpesvirus KSHV encodes a constitutively active G-protein-coupled receptor linked to cell proliferation. Nature 385: 347-350, 1997.

33. Bais C, Santomasso B, Coso O, et al: G-protein-coupled receptor of Kaposi's sarcoma-associated herpesvirus is a viral oncogene and angiogenesis activator. Nature 391: 86-89, 1998.

34. Boshoff C, Endo Y, Collins PD, et al: Angiogenic and HIV-inhibitory functions of KSHV-encoded chemokines. Science 278: 290-294, 1997.

35. Gao SJ, Boshoff C, Jayachandra S, Weiss RA, Chang Y and Moore PS: KSHV ORF K9 (vIRF) is an oncogene which inhibits the interferon signaling pathway. Oncogene 15: 1979-1985, 1997.

36. Lee H, Guo J, Li M, Choi JK, DeMaria M, Rosenzweig M and Jung JU: Identification of an immunoreceptor tyrosinebased activation motif of K1 transforming protein of Kaposi's sarcoma-associated herpesvirus. Mol Cell Biol 18: 5219-5228, 1998.

37. Sarid R, Sato T, Bohenzky RA, Russo JJ and Chang Y: Kaposi's sarcoma-associated herpesvirus encodes a functional bcl-2 homologue. Nat Med 3: 293-298, 1997.

38. Moore PS, Boshoff C, Weiss RA and Chang Y: Molecular mimicry of human cytokine and cytokine response pathway genes by KSHV. Science 274: 1739-1744, 1996.

39. Sun Q, Matta H, Lu G and Chaudhary PM: Induction of IL-8 expression by human herpesvirus 8 encoded vFLIP K13 via NF-kappaB activation. Oncogene 25: 2717-2726, 2006.

40. Osborne J, Moore PS and Chang Y: KSHV-encoded viral IL-6 activates multiple human IL-6 signaling pathways. Hum Immunol 60: 921-927, 1999.

41. Cesarman E, Moore PS, Rao PH, Inghirami G, Knowles DM and Chang Y: In vitro establishment and characterization of two acquired immunodeficiency syndrome-related lymphoma cell lines (BC-1 and BC-2) containing Kaposi's sarcoma-associated herpesvirus-like (KSHV) DNA sequences. Blood 86: 2708-2714, 1995.

42. Hu J, Garber AC and Renne R: The latency-associated nuclear antigen of Kaposi's sarcoma-associated herpesvirus supports latent DNA replication in dividing cells. J Virol 76: 11677-11687, 2002.

43. Jenner RG, Alba MM, Boshoff C and Kellam P: Kaposi's sarcoma-associated herpesvirus latent and lytic gene expression as revealed by DNA arrays. J Virol 75: 891-902, 2001. 
44. Pearce M, Matsumura S and Wilson AC: Transcripts encoding K12, v-FLIP, v-cyclin, and the microRNA cluster of Kaposi's sarcoma-associated herpesvirus originate from a common promoter. J Virol 79: 14457-14464, 2005.

45. Alkharsah KR, Singh VV, Bosco R, et al: Deletion of Kaposi's sarcoma-associated herpesvirus FLICE inhibitory protein, vFLIP, from the viral genome compromises the activation of STAT1-responsive cellular genes and spindle cell formation in endothelial cells. J Virol 85: 10375-10388, 2011.

46. Grossmann C, Podgrabinska S, Skobe M and Ganem D Activation of NF- $\kappa \mathrm{B}$ by the latent vFLIP gene of Kaposi's sarcoma-associated herpesvirus is required for the spindle shape of virus-infected endothelial cells and contributes to their proinflammatory phenotype. J Virol 80: 7179-7185, 2006.

47. Laemmli UK: Cleavage of structural proteins during the assembly of the head of bacteriophage T4. Nature 227: 680-685, 1970.

48. O'Farrell PH: High resolution two-dimensional electrophoresis of proteins. J Biol Chem 250: 4007-4021, 1975.

49. Klose J and Kobalz U: Two-dimensional electrophoresis of proteins: an updated protocol and implications for a functional analysis of the genome. Electrophoresis 16: 1034-1059, 1995.

50. Gorg A, Weiss W and Dunn MJ: Current two-dimensional electrophoresis technology for proteomics. Proteomics 4: 3665-3685, 2004.

51. Miller I, Crawford J and Gianazza E: Protein stains for proteomic applications: which, when, why? Proteomics 6: 5385-5408, 2006.

52. Neuhoff V, Arold N, Taube D and Ehrhardt W: Improved staining of proteins in polyacrylamide gels including isoelectric focusing gels with clear background at nanogram sensitivity using Coomassie Brilliant Blue G-250 and R-250. Electrophoresis 9: 255-262, 1988.

53. Berggren K, Chernokalskaya E, Steinberg TH, et al: Background-free, high sensitivity staining of proteins in oneand two-dimensional sodium dodecyl sulfate-polyacrylamide gels using a luminescent ruthenium complex. Electrophoresis 21: 2509-2521, 2000.

54. Berggren KN, Schulenberg B, Lopez MF, et al: An improved formulation of SYPRO Ruby protein gel stain: comparison with the original formulation and with a ruthenium II tris (bathophenanthroline disulfonate) formulation. Proteomics 2: 486-498, 2002.

55. Patton WF: Detection technologies in proteome analysis. J Chromatogr B Analyt Technol Biomed Life Sci 771: 3-31, 2002.

56. Chandramouli K and Qian PY: Proteomics: challenges, techniques and possibilities to overcome biological sample complexity. Hum Genom Proteomics 1: pii: 239204, 2009.

57. Unlu M, Morgan ME and Minden JS: Difference gel electrophoresis: a single gel method for detecting changes in protein extracts. Electrophoresis 18: 2071-2077, 1997.

58. May C, Brosseron F, Chartowski P, Schumbrutzki C, Schoenebeck B and Marcus K: Instruments and methods in proteomics. Methods Mol Biol 696: 3-26, 2011.

59. Michalski A, Cox J and Mann M: More than 100,000 detectable peptide species elute in single shotgun proteomics runs but the majority is inaccessible to data-dependent LC-MS/MS. J Proteome Res 10: 1785-1793, 2011.

60. Washburn MP, Wolters D and Yates JR III: Large-scale analysis of the yeast proteome by multidimensional protein identification technology. Nat Biotechnol 19: 242-247, 2001.

61. Yates JR III, Eng JK, McCormack AL and Schieltz D: Method to correlate tandem mass spectra of modified peptides to amino acid sequences in the protein database. Anal Chem 67: 1426-1436, 1995.

62. Munday DC, Surtees R, Emmott E, et al: Using SILAC and quantitative proteomics to investigate the interactions between viral and host proteomes. Proteomics 12: 666-672, 2012.

63. Ong SE, Blagoev B, Kratchmarova I, Kristensen DB, Steen H, Pandey A and Mann M: Stable isotope labeling by amino acids in cell culture, SILAC, as a simple and accurate approach to expression proteomics. Mol Cell Proteomics 1: 376-386, 2002.

64. Krijgsveld J, Ketting RF, Mahmoudi T, et al: Metabolic labeling of C. elegans and D. melanogaster for quantitative proteomics. Nat Biotechnol 21: 927-931, 2003.

65. Kruger M, Moser M, Ussar S, et al: SILAC mouse for quantitative proteomics uncovers kindlin-3 as an essential factor for red blood cell function. Cell 134: 353-364, 2008.

66. Ong SE, Kratchmarova I and Mann M: Properties of ${ }^{13} \mathrm{C}$-substituted arginine in stable isotope labeling by amino acids in cell culture (SILAC). J Proteome Res 2: 173-181, 2003.
67. Gygi SP, Rist B, Gerber SA, Turecek F, Gelb $\mathrm{MH}$ and Aebersold R: Quantitative analysis of complex protein mixtures using isotope-coded affinity tags. Nat Biotechnol 17: 994-999, 1999.

68. Schmidt A, Kellermann J and Lottspeich F: A novel strategy for quantitative proteomics using isotope-coded protein labels. Proteomics 5: 4-15, 2005.

69. DeSouza L, Diehl G, Rodrigues MJ, Guo J, Romaschin AD, Colgan TJ and Siu KW: Search for cancer markers from endometrial tissues using differentially labeled tags iTRAQ and cICAT with multidimensional liquid chromatography and tandem mass spectrometry. J Proteome Res 4: 377-386, 2005.

70. Liu H, Sadygov RG and Yates JR III: A model for random sampling and estimation of relative protein abundance in shotgun proteomics. Anal Chem 76: 4193-4201, 2004

71. Bantscheff M, Schirle M, Sweetman G, Rick J and Kuster B: Quantitative mass spectrometry in proteomics: a critical review. Anal Bioanal Chem 389: 1017-1031, 2007.

72. Zhang Y, Wen Z, Washburn MP and Florens L: Refinements to label free proteome quantitation: how to deal with peptides shared by multiple proteins. Anal Chem 82: 2272-2281, 2010.

73. Florens L, Carozza MJ, Swanson SK, Fournier M, Coleman MK, Workman JL and Washburn MP: Analyzing chromatin remodeling complexes using shotgun proteomics and normalized spectral abundance factors. Methods 40: 303-311, 2006.

74. Zybailov B, Mosley AL, Sardiu ME, Coleman MK, Florens L and Washburn MP: Statistical analysis of membrane proteome expression changes in Saccharomyces cerevisiae. J Proteome Res 5: 2339-2347, 2006.

75. Lu P, Vogel C, Wang R, Yao X and Marcotte EM: Absolute protein expression profiling estimates the relative contributions of transcriptional and translational regulation. Nat Biotechnol 25: 117-124, 2007.

76. Bondarenko PV, Chelius D and Shaler TA: Identification and relative quantitation of protein mixtures by enzymatic digestion followed by capillary reversed-phase liquid chromatography-tandem mass spectrometry. Anal Chem 74: 4741-4749, 2002.

77. Chelius D and Bondarenko PV: Quantitative profiling of proteins in complex mixtures using liquid chromatography and mass spectrometry. J Proteome Res 1: 317-323, 2002.

78. Wong JW and Cagney G: An overview of label-free quantitation methods in proteomics by mass spectrometry. Methods Mol Biol 604: 273-283, 2010.

79. Tate S, Larsen B, Bonner R and Gingras AC: Label-free quantitative proteomics trends for protein-protein interactions. J Proteomics 81: 91-101, 2013.

80. Li Z, Adams RM, Chourey K, Hurst GB, Hettich RL and Pan C: Systematic comparison of label-free, metabolic labeling, and isobaric chemical labeling for quantitative proteomics on LTQ Orbitrap Velos. J Proteome Res 11: 1582-1590, 2012.

81. Lippe R: Deciphering novel host-herpesvirus interactions by virion proteomics. Front Microbiol 3: 181, 2012.

82. Johannsen E, Luftig M, Chase MR, et al: Proteins of purified Epstein-Barr virus. Proc Natl Acad Sci USA 101: 16286-16291, 2004.

83. Zhu FX, Chong JM, Wu L and Yuan Y: Virion proteins of Kaposi's sarcoma-associated herpesvirus. J Virol 79: 800-811, 2005.

84. Gould F, Harrison SM, Hewitt EW and Whitehouse A: Kaposi's sarcoma-associated herpesvirus RTA promotes degradation of the Heyl repressor protein through the ubiquitin proteasome pathway. J Virol 83: 6727-6738, 2009.

85. Alsayed Y, Leleu X, Leontovich A, Oton AB, Malhem M, George D and Ghobrial IM: Proteomics analysis in post-transplant lymphoproliferative disorders. Eur J Haematol 81: 298-303, 2008.

86. Sun X, Barlow EA, Ma S, et al: Hsp90 inhibitors block outgrowth of EBV-infected malignant cells in vitro and in vivo through an EBNA1-dependent mechanism. Proc Natl Acad Sci USA 107: 3146-3151, 2010.

87. Sun X and Kenney SC: Hsp90 inhibitors: a potential treatment for latent EBV infection? Cell Cycle 9: 1665-1666, 2010.

88. Meckes DG Jr and Raab-Traub N: Microvesicles and viral infection. J Virol 85: 12844-12854, 2011.

89. Pegtel DM, Cosmopoulos K, Thorley-Lawson DA, et al: Functional delivery of viral miRNAs via exosomes. Proc Natl Acad Sci USA 107: 6328-6333, 2010.

90. Raimondo F, Morosi L, Chinello C, Magni F and Pitto M: Advances in membranous vesicle and exosome proteomics improving biological understanding and biomarker discovery. Proteomics 11: 709-720, 2011. 
91. Meckes DG Jr, Shair KH, Marquitz AR, Kung CP, Edwards RH and Raab-Traub N: Human tumor virus utilizes exosomes for intercellular communication. Proc Natl Acad Sci USA 107: 20370-20375, 2010

92. Meckes DG Jr, Gunawardena HP, Dekroon RM, et al: Modulation of B-cell exosome proteins by gamma herpesvirus infection. Proc Natl Acad Sci USA 110: E2925-E2933, 2013

93. Schlee M, Krug T, Gires O, et al: Identification of EpsteinBarr virus (EBV) nuclear antigen 2 (EBNA2) target proteins by proteome analysis: activation of EBNA2 in conditionally immortalized B cells reflects early events after infection of primary B cells by EBV. J Virol 78: 3941-3952, 2004.

94. Thurau M, Marquardt G, Gonin-Laurent N, et al: Viral inhibitor of apoptosis vFLIP/K13 protects endothelial cells against superoxide-induced cell death. J Virol 83: 598-611, 2009.

95. Sakakibara S, Pise-Masison CA, Brady JN and Tosato G: Gene regulation and functional alterations induced by Kaposi's sarcoma-associated herpesvirus-encoded ORFK13/vFLIP in endothelial cells. J Virol 83: 2140-2153, 2009.

96. Feng X, Zhang J, Chen WN and Ching CB: Proteome profiling of Epstein-Barr virus infected nasopharyngeal carcinoma cell line: identification of potential biomarkers by comparative iTRAQ-coupled 2D LC/MS-MS analysis. J Proteomics 74 567-576, 2011

97. Bartee E, McCormack A and Früh K: Quantitative membrane proteomics reveals new cellular targets of viral immune modulators. PLoS Pathog 2: e107, 2006.

98. Si H, Verma SC and Robertson ES: Proteomic analysis of the Kaposi's sarcoma-associated herpesvirus terminal repeat element binding proteins. J Virol 80: 9017-9030, 2006.

99. Kaul R, Verma SC and Robertson ES: Protein complexes associated with the Kaposi's sarcoma-associated herpesvirus-encoded LANA. Virology 364: 317-329, 2007.

100. Boyne JR, Jackson BR, Taylor A, Macnab SA and Whitehouse A: Kaposi's sarcoma-associated herpesvirus ORF57 protein interacts with PYM to enhance translation of viral intronless mRNAs. EMBO J 29: 1851-1864, 2010.

101. Jackson BR, Boyne JR, Noerenberg M, et al: An interaction between KSHV ORF57 and UIF provides mRNA-adaptor redundancy in Herpesvirus intronless mRNA export. PLoS Pathog 7: e1002138, 2011.

102. Hiscox JA, Whitehouse A and Matthews DA: Nucleolar proteomics and viral infection. Proteomics 10: 4077-4086, 2010.

103. Boyne JR and Whitehouse A: Nucleolar disruption impairs Kaposi's sarcoma-associated herpesvirus ORF57-mediated nuclear export of intronless viral mRNAs. FEBS Lett 583 3549-3556, 2009.

104. Taylor A, Jackson BR, Noerenberg M, et al: Mutation of a C-terminal motif affects Kaposi's sarcoma-associated herpesvirus ORF57 RNA binding, nuclear trafficking, and multimerization. J Virol 85: 7881-7891, 2011.

105. Jackson BR, Noerenberg Mand Whitehouse A: A novel mechanism inducing genome instability in Kaposi's sarcoma-associated herpesvirus infected cells. PLoS Pathog 10: e1004098, 2014.

106. Malik-Soni N and Frappier L: Proteomic profiling of EBNA1-host protein interactions in latent and lytic Epstein-Barr virus infections. J Virol 86: 6999-7002, 2012.

107. Howe JG and Shu MD: Isolation and characterization of the genes for two small RNAs of herpesvirus papio and their comparison with Epstein-Barr virus-encoded EBER RNAs. J Virol 62: 2790-2798, 1988.

108. Szebeni A, Mehrotra B, Baumann A, Adam SA, Wingfield PT and Olson MO: Nucleolar protein B23 stimulates nuclear import of the HIV-1 Rev protein and NLS-conjugated albumin. Biochemistry 36: 3941-3949, 1997.

109. Sander G, Konrad A, Thurau M, et al: Intracellular localization map of human herpesvirus 8 proteins. J Virol 82: 1908-1922, 2008

110. Labo N, Miley W, Marshall V, et al: Heterogeneity and breadth of host antibody response to KSHV infection demonstrated by systematic analysis of the KSHV proteome. PLoS Pathog 10 e1004046, 2014.
111. Evans VC, Barker G, Heesom KJ, Fan J, Bessant C and Matthews DA: De novo derivation of proteomes from transcriptomes for transcript and protein identification. Nat Methods 9: 1207-1211, 2012

112. Dresang LR, Teuton JR, Feng H, et al: Coupled transcriptome and proteome analysis of human lymphotropic tumor viruses: insights on the detection and discovery of viral genes. BMC Genomics 12: 625, 2011

113. Couzin J: Breakthrough of the year. Small RNAs make big splash. Science 298: 2296-2297, 2002.

114. Dennis C: Small RNAs: the genome's guiding hand? Nature 420: 732,2002

115. Jopling CL: Regulation of hepatitis C virus by microRNA-122. Biochem Soc Trans 36: 1220-1223, 2008.

116. Ou M, Zhang X, Dai Y, et al: Identification of potential microRNA-target pairs associated with osteopetrosis by deep sequencing, iTRAQ proteomics and bioinformatics. Eur J Hum Genet 5: 625-632, 2013 .

117. Gallaher AM, Das S, Xiao Z, Andresson T, Kieffer-Kwon P, Happel $\mathrm{C}$ and Ziegelbauer J: Proteomic screening of human targets of viral microRNAs reveals functions associated with immune evasion and angiogenesis. PLoS Pathog 9: e1003584, 2013.

118. Huang TC, Pinto SM and Pandey A: Proteomics for understanding miRNA biology. Proteomics 13: 558-567, 2013

119. Wu S, Li N, Ma J, et al: First proteomic exploration of protein-encoding genes on chromosome 1 in human liver, stomach, and colon. J Proteome Res 12: 67-80, 2013.

120. Zhang C, Li N, Zhai L, et al: Systematic analysis of missing proteins provides clues to help define all of the protein-coding genes on human chromosome 1. J Proteome Res 13: 114-125, 2013.

121. Tang F, Barbacioru C, Wang Y, et al: mRNA-Seq whole-transcriptome analysis of a single cell. Nat Methods 6: 377-382, 2009.

122. Islam S, Kjallquist U, Moliner A, Zajac P, Fan JB, Lönnerberg P and Linnarsson S: Highly multiplexed and strand-specific single-cell RNA 5' end sequencing. Nat Protoc 7: 813-828, 2012.

123. Salehi-Reyhani A, Kaplinsky J, Burgin E, et al: A first step towards practical single cell proteomics: a microfluidic antibody capture chip with TIRF detection. Lab Chip 11: 1256-1261, 2011.

124. Lamond A: http://www.peptracker.com/dm/. Accessed June 1, 2014.

125. Boisvert FM, Ahmad Y, Gierliński M, et al: A quantitative spatial proteomics analysis of proteome turnover in human cells. Mol Cell Proteomics 11: M111.011429, 2012.

126. von Mering C, Huynen M, Jaeggi D, Schmidt S, Bork P and Snel B: STRING: a database of predicted functional associations between proteins. Nucleic Acids Res 31: 258-261, 2003

127. Mann M, Aebersold R, Robinson CV, et al: http://www.propsects-fp7.eu/resources/index.html. Accessed June 1,2014.

128. Krupp M, Marquardt JU, Sahin U, Galle PR, Castle J and Teufel A: RNA-Seq Atlas - a reference database for gene expression profiling in normal tissue by next-generation sequencing. Bioinformatics 28: 1184-1185, 2012.

129. Vizcaino JA, Deutsch EW, Wang R, et al: ProteomeXchange provides globally coordinated proteomics data submission and dissemination. Nat Biotechnol 32: 223-226, 2014.

130. Vinayagam A, Zirin J, Roesel C, et al: Integrating protein-protein interaction networks with phenotypes reveals signs of interactions. Nat Methods 11: 94-99, 2014.

131. Jones JF, Shurin S, Abramowsky C, et al: T-cell lymphomas containing Epstein-Barr viral DNA in patients with chronic Epstein-Barr virus infections. New Eng1 J Med 318: 733-741, 1988.

132. Old LJ, Boyse EA, Oettgen HF, De Harven E, Geering G, Williamson B and Clifford P: Precipitating antibody in human serum to an antigen present in cultured Burkitt's lymphoma cells. Proc Natl Acad Sci USA 56: 1699-1704, 1966. 